\title{
Factores asociados a la alteración de la glicemia basal en el primer control posterior a una hospitalización en pacientes con diabetes mellitus tipo 2
}

Ana Denisse Alva Cabrera 1,a, Wilfor Aguirre Quispe 2,b, Carlos Alexander Alva Díaz 1,c, Jorge Alonso García Mostajo ${ }^{3, d}$, Alexander Amilcar Zapana Mansilla ${ }^{1, a}$

\section{RESUMEN}

Objetivo: Analizar los factores asociados a la alteración de la glicemia basal en el primer control posterior a una hospitalización en pacientes con DM2.

Materiales y métodos: Se realizó un estudio observacional, retrospectivo, de casos y controles, en proporción 1:1. Se analizaron 100 registros médicos de pacientes con DM2, de ambos sexos, mayores de 18 años, con un control médico dentro de los 3 primeros meses posteriores a la hospitalización. Los casos definidos mediante glicemia basal elevada (> $130 \mathrm{mg} / \mathrm{dL}$ ) y los controles definidos como glicemia normal ( $\leq 130 \mathrm{mg} / \mathrm{dL})$. Se calculó medias y porcentajes, se usó la prueba $\mathrm{Chi}^{2}$ y regresión logística binaria.

Resultados: Niveles elevados de glicemia al ingreso (OR: 2.7, $\mathrm{p}<0.05)$, niveles elevados de glicemia al alta (OR: 3.7, $\mathrm{p}<$ $0.005), \mathrm{HbA} 1 \mathrm{c} \geq 9 \%$ durante la hospitalización (OR: 2.9, $\mathrm{p}<0.05$ ), niveles elevados de triglicéridos (OR: 2.6, $\mathrm{p}<0.05)$ y no cumplimiento del tratamiento prescrito (OR: 2.15, $\mathrm{p}<0.005$ ) se asociaron a una glicemia basal elevada en el primer control post hospitalización.

Conclusiones: Los niveles elevados de triglicéridos y el incumplimiento de indicaciones son factores de riesgo asociados a un pobre control glicémico en la primera consulta externa luego de la hospitalización.

Palabras clave: Diabetes mellitus; glucemia; hospitalización (Fuente: DeCS BIREME).

\section{Factors associated with the alteration of baseline glycemia in the first post- hospitalization checkup in patients with type 2 diabetes mellitus}

\section{ABSTRACT}

Objective: To analyze the factors associated with the alteration of baseline glycemia in the first post-hospitalization checkup in patients with type 2 diabetes mellitus (T2DM)

Materials and methods: A retrospective observational case-control study was performed in a 1:1 ratio. One hundred (100) medical records of patients of both genders and over 18 years of age with T2DM who had a medical checkup within the first 3 months after hospitalization were analyzed. Cases were defined as those with high baseline glycemia (> 130 $\mathrm{mg} / \mathrm{dL}$ ) and controls were defined as those who had normal glycemic levels $(\leq 130 \mathrm{mg} / \mathrm{dL})$. Mean and percentages were estimated using the chi-square test and binary logistic regression.

Results: Elevated glycemic levels at admission (OR: 2.7, $p<0.05)$, elevated glycemic levels at discharge (OR: 3.7, $p$ $<0.005$ ), HbA1c $\geq 9 \%$ during hospitalization (OR: 2.9, p <0.05), high levels of triglycerides (OR: $2.6, p<0.05)$, and failure to comply with prescribed treatment (OR: 2.15, $\mathrm{p}<0.005)$ were associated with high baseline glycemia in the first posthospitalization checkup.

Conclusions: High levels of triglycerides and non-compliance with indications are risk factors associated with poor glycemic control at the first outpatient visit after hospitalization.

Keywords: Diabetes mellitus; blood glucose; hospitalization (Source: MeSH NLM).

1. Hospital Daniel Alcides Carrión. Lima, Perú.

2. Instituto Nacional de Salud. Lima, Perú.

3. Hospital Guillermo Kaelin de la Fuente. Lima, Perú.

a. Médico Endocrinólogo.

b. Médico Cirujano.

c. Médico Neurólogo.

d. Médico Internista. 


\section{INTRODUCCIÓN}

La diabetes mellitus tipo 2 (DM2) es una enfermedad crónica con una elevada tasa de morbimortalidad debido a las complicaciones que produce, las cuales requieren de atención médica continua, generando un impacto negativo en la calidad de vida del paciente y de su entorno ${ }^{(1,2,3)}$.

Según la Federación Internacional de Diabetes (IDF) (4), a nivel mundial existen 415 millones de adultos con diagnóstico de DM2 con una prevalencia que oscila entre $7.2 \%-11.4 \%$, y se predice un aumento marcado de personas que desarrollarán la enfermedad para el 2040, aumentando a 642 millones. A nivel del Perú la estimación más reciente muestra que la incidencia acumulada de DM2 es de $7,2 \%$ con una tasa de incidencia de 19,5 casos nuevos por 1000 personas-año ${ }^{(5)}$.

Asimismo, se ha reportado que casi un tercio de los pacientes con DM2 requiere 2 o más hospitalizaciones en un año determinado y que incluso el $75 \%$ de pacientes con DM2 necesitarán ser hospitalizados en algún momento de sus vidas ${ }^{(6,7)}$. En el Perú los datos brindados por el Ministerio de Salud señalan a la DM2 como la décimoctava patología causante de hospitalización a nivel nacional durante el año 2015 con una tasa del 0.9 $\%$ de la población total hospitalizada ${ }^{(8)}$.

Aunque existen distintas organizaciones que han elaborado directrices a seguir sobre los objetivos de glicemia tanto en el ámbito hospitalario como ambulatorio ${ }^{(9,10)}$, la Asociación Americana de Diabetes establece como objetivo de adecuado control glicémico en el área extra hospitalaria a una glicemia $\leq 130 \mathrm{mg} / \mathrm{dL}$ en ayunas, sin embargo estos parámetros establecidos son muchas veces difíciles de alcanzar por diversos factores ${ }^{(10,11)}$.

Las posibles causas de no lograr los objetivos recomendados son múltiples y pueden deberse a elementos relacionados con la enfermedad y su evolución, pero también con factores externos como la actitud de los médicos y pacientes en relación con el tratamiento de la enfermedad (12). Es por ello que la transición del paciente hospitalizado a la atención ambulatoria es fundamental en el manejo del paciente con DM2 y la identificación de los factores que pueden contribuir a una alteración de la glicemia basal en el primer control posthospitalización resulta necesaria para desarrollar estrategias destinadas a mejorar el cumplimiento de las metas establecidas.

El objetivo del presente estudio fue evaluar los posibles factores de riesgo asociados a la alteración de la glicemia basal en el primer control por consultorio externo posterior a una hospitalización en paciente con DM2, a partir de una población de pacientes del Hospital Nacional Daniel Alcides Carrión.

\section{MATERIALES Y METODOS}

Se realizó un estudio observacional, retrospectivo, con un diseño de casos y controles. Se revisaron los registros médicos de pacientes en su primera consulta posterior al alta de una hospitalización por DM2 descompensada, en el Hospital Nacional Daniel Alcides Carrión de la ciudad de Lima.

Se tomó como población de análisis los registros de pacientes con DM2 mayores de 18 años, de ambos sexos, que se hospitalizaron en los Servicios de Endocrinología y Medicina Interna del Hospital Daniel Alcides Carrión debido a una descompensación de la enfermedad, en el período comprendido entre el 1 de enero y el 31 de diciembre del 2014, y que acudieron a control médico por consultorio externo dentro de los 3 primeros meses posteriores al alta. Se excluyeron a aquellos pacientes cuyo motivo de ingreso fue hipoglicemia.

El estudio tuvo un muestreo no probabilístico, por conveniencia. Se calculó el tamaño muestral para comparación de proporciones, necesitándose un tamaño muestral mínimo de 50 pacientes por grupo. El cálculo del tamaño muestral consideró un nivel de significancia del $5 \%$ y una potencia estadística mínima del $80 \%$, se utilizaron los datos aportados por el estudio de Khattab $M$. et al ${ }^{12}$. Luego de aplicar los criterios de selección se obtuvieron 121 registros de pacientes que ingresaron por consultorio externo dentro del período establecido, de estos, se consideró glicemia basal normal a quienes presentaron glicemias $\leq 130 \mathrm{mg} / \mathrm{dL}$ en su primera evaluación médica posterior a la hospitalización (grupo control), y alteración de la glicemia basal quienes presentaron glicemia $>130 \mathrm{mg} / \mathrm{dL}$ (grupo de casos). Teniendo en cuenta la muestra mínima necesaria para lograr una proporción 1:1 entre casos y controles, se consideró 50 pacientes por grupo (Figura 1). 


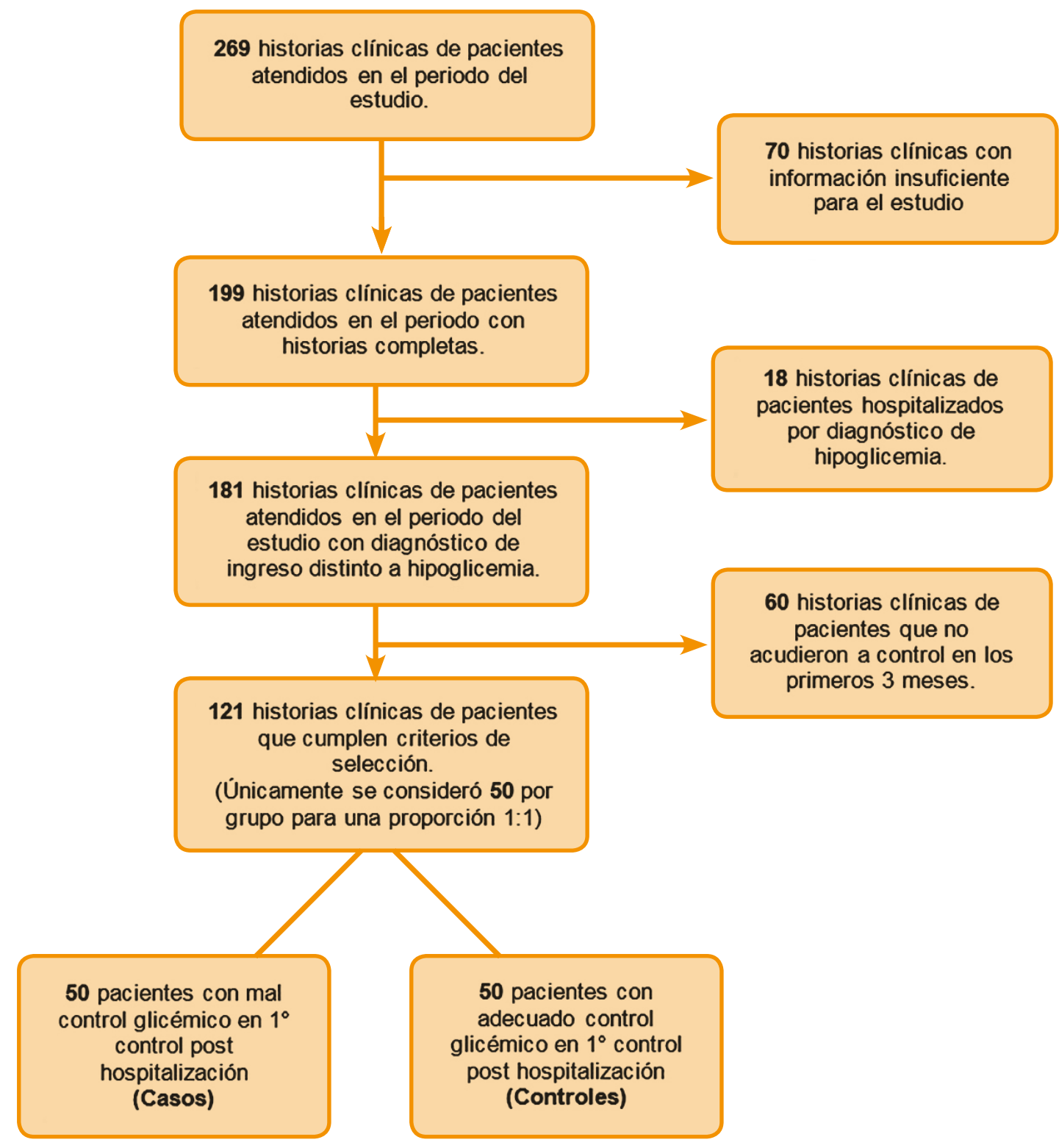

Figura 1. Flujograma del estudio para selección de datos

Las variables de interés fueron aquellos potenciales factores de riesgo para el desarrollo de un mal control glicémico después de que el paciente fue dado de alta y previo a la primera consulta médica post-hospitalización.

Estas variables fueron, tanto epidemiológicas: sexo y edad (18-40, 41-64, $\geq 65$ años), como clínicas: peso, tiempo de la enfermedad, motivo de hospitalización, niveles de glicemia al ingreso y al alta, porcentajes de hemoglobina glicosilada durante la hospitalización (HbA1c), dislipidemia, niveles de lipoproteína de baja densidad (LDL), niveles de triglicéridos, hipertensión arterial (HTA), tasa de filtración glomerular (TFG), complicaciones crónicas, días de hospitalización, medicación previa a la hospitalización y al alta, tipo de medicación al alta (antidiabéticos orales vs insulina sola o combinada con antidiabéticos orales), primer control por consultorio externo (mayor o menor a 30 días), cumplimiento del tratamiento (sí/no) y servicio médico donde fue hospitalizado (Endocrinología o Medicina). La dislipidemia se definió cómo la presencia de 
al menos un valor elevado de colesterol LDL ( $\geq 100 \mathrm{mg} / \mathrm{dL}$ ) o triglicéridos ( $\geq 150 \mathrm{mg} / \mathrm{dL}$ ), lamentablemente, los datos de las lipoproteína de alta densidad (HDL) no se incluyeron en el estudio porque en muchos de los pacientes incluidos no se consignó el resultado en la historia clínica por diversas razones.

Respecto a la variable niveles de glicemia al ingreso a hospitalización, se categorizó en $\geq 250 \mathrm{mg} / \mathrm{dL}$ y $<250$ $\mathrm{mg} / \mathrm{dL}$, por ser el parámetro bioquímico para considerar una crisis hiperglicémica, la que puede derivar en una emergencia como es la cetoacidosis diabética ${ }^{(13)}$. Los niveles de glicemia al alta se categorizaron en 2 grupos, $67-139 \mathrm{mg} / \mathrm{dL}$ y $\geq 140 \mathrm{mg} / \mathrm{dL}$, ya que dicho punto de corte es el objetivo máximo de glicemia del paciente con DM2 hospitalizado ${ }^{(10)}$.

En relación al punto de corte de la variable $\mathrm{HbA1c}$, aunque se sabe que el objetivo de buen control glicémico establecido por las diferentes sociedades de DM2 es una $\mathrm{HbA1C}$ de $<7 \%^{(2,10)}$, se reconoce que es necesario tener en cuenta la edad, expectativa de vida, antecedentes de hipoglicemias graves, comorbilidad, existencia de complicaciones y presencia de otros factores de riesgo cardiovascular, en cuyos caso el objetivo de HbA1c suele ser mayor ${ }^{(10)}$, y para tratar de estandarizar esto, el estudio consideró un punto de corte de $9 \%$, además de existir evidencia que con una HbA1c > $9 \%$ es necesario el inicio de terapia intensiva en el paciente con DM2 ${ }^{(14)}$, esquema que la mayoría de pacientes del estudio recibieron durante la hospitalización. Las demás variables fueron categorizadas de acuerdo a puntos de corte establecidos según se muestra en la Tabla 1.

\section{Procedimiento estadístico}

El análisis univariado para las variables de tipo cualitativa o categórica se realizó mediante cálculo de porcentajes y las variables de tipo numérica o cuantitativa mediante medias y desviación estándar (DE). El análisis bivariado para variables de naturaleza cualitativa se realizó mediante la prueba estadística no paramétrica $\mathrm{Chi}^{2}$ y la corrección de Yates cuando fue requerido. El análisis multivariado empleado fue la regresión logística binaria, se empleó el método de estimación por pasos mediante la adición de variables significativas según análisis bivariado, se calculó el $\mathrm{Chi}^{2}$ de Wald. La información fue recolectada en una base de datos electrónica y analizada en el programa estadístico Stata V14.

\section{Aspectos éticos}

El presente trabajo se ejecutó con estricto respeto de los principios éticos establecidos en las normas internacionales de investigación (Declaración de Helsinki). Por ser una investigación observacional tipo retrospectivo, los pacientes no fueron sujetos de intervenciones adicionales. Se solicitó la autorización de revisión de historias clínicas a la Oficina de Docencia e Investigación y a la Oficina de Estadística del Hospital Nacional Daniel Alcides Carrión.

\section{RESULTADOS}

Este estudio incluyó a un total de 100 pacientes (50 casos y 50 controles) con DM2 con edades comprendidas entre 23 y 94 años, con una media de 57,6 años (DE: \pm 12.9 ), y un peso medio de 69.4 kilos (D.E.: \pm 14.8 ).

El conjunto de las características clínicas se muestran detalladamente en la tabla 1. Las causas de hospitalización previa al control por consultorio de los pacientes fueron en similares proporciones de etiología infecciosa y crisis por hiperglicemia, el $35 \%$; mientras que una menor proporción fue por pie diabético, $14 \%$, y otras causas, $16 \%$. Durante su hospitalización, previa al control por consultorio, los niveles de glicemia de ingreso fueron mayores de 250 $\mathrm{mg} / \mathrm{dL}$ en $56 \%$ de pacientes y la glicemia al alta fue $\geq$ $140 \mathrm{mg} / \mathrm{dL}$ en $49 \%$ de los pacientes; también se encontró una HbA1c $\geq 9 \%$ en un $67.4 \%$ de los pacientes durante su hospitalización. En cuanto al perfil lipídico de los pacientes se encontró que presentaron dislipidemia el $95.6 \%$, niveles de $\mathrm{LDL} \geq 100 \mathrm{mg} / \mathrm{dL}$ el $47.7 \%$, triglicéridos $\geq 150 \mathrm{mg} / \mathrm{dL}$ el $46.7 \%$. Los pacientes además presentaron el diagnóstico de HTA en $40.4 \%$ y niveles de TFG $<60 \mathrm{ml} /$ $\mathrm{min} / 1.73 \mathrm{~m}^{2}$ en el $22.2 \%$. La duración de la hospitalización fue menor de 14 días en el $68 \%$ de los pacientes.

El $56 \%$ de los pacientes recibían algún tipo de tratamiento antes de la hospitalización y $90 \%$ después de la hospitalización. En cuanto al tipo de tratamiento al alta, al $85.6 \%$ de los pacientes usaron insulina sola o insulina asociado a algún antidiabético oral, mientras que el $14.4 \%$ de los pacientes usaron únicamente algún tipo de antidiabético oral. Un $74 \%$ de los pacientes regresaron a su control antes de los 30 días después de su hospitalización y el 86 \% si cumplió el tratamiento prescrito al alta. El $61 \%$ de los pacientes estuvieron hospitalizados en el Servicio de Endocrinología y el 39 \% en el Servicio de Medicina.

En el análisis bivariado, las características que se asociaron a la alteración de la glicemia basal en el primer control posterior a una hospitalización, en pacientes con DM2, fueron los niveles elevados de glicemia al ingreso (OR: 2.7, $\mathrm{p}<0.05$ ), los niveles de glicemia al alta (OR: 3.7, $\mathrm{p}<$ 0.005 ), los niveles de HbA1c $\geq 9 \%$ (OR: $2.9, p<0.05$ ), los niveles elevados de triglicéridos (OR: $2.6, p<0.05$ ), y no cumplir con el tratamiento prescrito (OR: 2.15, $p<0.005$ ). El tratamiento a base de insulina sola o en combinación presentó un valor $p$ límite pero su condición de riesgo se confirmó en el intervalo de confianza del OR (OR: 1.6, IC: 1.03 - 2.52) (Tabla 1). 
Tabla 1. Proporción de pacientes con alteración de la glicemia basal en el primer control posterior a una hospitalización en pacientes con diabetes mellitus tipo 2 , según las características epidemiológicas y clínicas

\begin{tabular}{|c|c|c|c|c|c|c|c|c|}
\hline \multirow[t]{2}{*}{ Variable } & \multicolumn{2}{|c|}{$\begin{array}{l}\text { Glicemia } \\
\text { elevada }\end{array}$} & \multicolumn{2}{|c|}{$\begin{array}{c}\text { Glicemia } \\
\text { normal }\end{array}$} & \multicolumn{2}{|c|}{ Total } & \multirow[t]{2}{*}{$p\left({ }^{*}\right)$} & \multirow[t]{2}{*}{ OR (IC 95) } \\
\hline & $\mathbf{n}$ & $\%$ & $\mathbf{n}$ & $\%$ & $\mathbf{n}$ & $\%$ & & \\
\hline \multicolumn{9}{|l|}{ Sexo } \\
\hline Masculino & 20 & 46,5 & 23 & 53,5 & 43 & 43 & \multirow{2}{*}{0,54} & $0,78(0,32-1,86)$ \\
\hline Femenino & 30 & 52,6 & 27 & 47,4 & 57 & 57 & & $\ldots$ \\
\hline \multicolumn{9}{|l|}{ Edad } \\
\hline $18-40$ años & 11 & 68,8 & 5 & 31,2 & 16 & 16 & 0,17 & $2,5(0,72-10,1)$ \\
\hline 41-64 años & 27 & 46,6 & 31 & 53,4 & 58 & 58 & 0,54 & $0,71(0,30-1,71)$ \\
\hline$\geq 65$ años & 12 & 46,2 & 14 & 53,8 & 26 & 26 & 0,82 & $0,81(0,29-2,18)$ \\
\hline \multicolumn{9}{|l|}{ Tiempo de enfermedad } \\
\hline$<10$ años & 36 & 45,6 & 43 & 54,4 & 79 & 79 & \multirow{2}{*}{0,086} & $0,41(0,12-1,26)$ \\
\hline$\geq 10$ años & 14 & 66,7 & 7 & 33,3 & 21 & 21 & & $\ldots$ \\
\hline \multicolumn{9}{|l|}{ Motivo de hospitalización } \\
\hline Infecciones & 17 & 48,6 & 18 & 51,4 & 35 & 35 & 0,83 & $0,91(0,37-2,25)$ \\
\hline Crisis hiperglicémica & 16 & 45,7 & 19 & 54,3 & 35 & 35 & 0,52 & $0,76(0,30-1,89)$ \\
\hline Pie diabético & 10 & 71,4 & 4 & 28,6 & 14 & 14 & 0,08 & $2,87(0,74-13,4)$ \\
\hline Otras causas & 7 & 43,8 & 9 & 56,2 & 16 & 16 & $\ldots$ & $\ldots$ \\
\hline \multicolumn{9}{|c|}{ Glicemia al ingreso en hospitalización } \\
\hline $74-250 \mathrm{mg} / \mathrm{dL}$ & 16 & 36,4 & 28 & 63,6 & 44 & 44 & \multirow{2}{*}{0,016} & $\ldots$ \\
\hline$\geq 250 \mathrm{mg} / \mathrm{dL}$ & 34 & 60,7 & 22 & 39,4 & 56 & 56 & & $2,7(1,11-6,64)$ \\
\hline \multicolumn{9}{|c|}{ Glicemia al alta de hospitalización } \\
\hline $67-139 \mathrm{mg} / \mathrm{dL}$ & 18 & 35,3 & 33 & 64,7 & 51 & 51 & \multirow{2}{*}{0,003} & $\ldots$ \\
\hline$\geq 140 \mathrm{mg} / \mathrm{dL}$ & 32 & 65,3 & 17 & 34,7 & 49 & 49 & & $3,45(1,40-8,54)$ \\
\hline \multicolumn{9}{|c|}{ HbA1c durante la hospitalización } \\
\hline $4.6-8.9 \%$ & 9 & 31 & 20 & 69 & 29 & 32,6 & \multirow{2}{*}{0,023} & $\ldots$ \\
\hline$\geq 9 \%$ & 34 & 56,7 & 26 & 43,3 & 60 & 67,4 & & $2,9(1,04-8,42)$ \\
\hline \multicolumn{9}{|l|}{ Dislipidemia } \\
\hline Sí & 41 & 47,7 & 45 & 52,3 & 86 & 95,6 & \multirow{2}{*}{0,37} & $2,7(0,20-146,9)$ \\
\hline No & 1 & 25 & 3 & 75 & 4 & 4,4 & & $\ldots$ \\
\hline \multicolumn{9}{|l|}{ LDL } \\
\hline $28.6-99 \mathrm{mg} / \mathrm{dL}$ & 19 & 42,2 & 26 & 57,8 & 45 & 52,3 & \multirow{2}{*}{0,4} & $\ldots$ \\
\hline$\geq 100 \mathrm{mg} / \mathrm{dL}$ & 21 & 51,2 & 20 & 48,8 & 41 & 47,7 & & $1,43(0,56-3,67)$ \\
\hline \multicolumn{9}{|l|}{ Triglicéridos } \\
\hline $51-149 \mathrm{mg} / \mathrm{dL}$ & 17 & 35,4 & 31 & 64,6 & 48 & 53,3 & 0.022 & $\ldots$ \\
\hline$\geq 150 \mathrm{mg} / \mathrm{dL}$ & 25 & 59,6 & 17 & 40,5 & 42 & 46,7 & & $2,68(1,05-6,89)$ \\
\hline HTA & & & & & & & & \\
\hline Sí & 21 & 52,5 & 19 & 47,5 & 40 & 40,4 & 074 & $1,14(0,47-2,76)$ \\
\hline No & 29 & 49,2 & 30 & 50,8 & 59 & 59,6 & 0,14 & $\ldots$ \\
\hline Tasa de filtración glome & & & & & & & & \\
\hline$<60 \mathrm{ml} / \mathrm{min} / 1.73 \mathrm{~m}^{2}$ & 9 & 45 & 11 & 55 & 20 & 22,2 & 063 & $0,61(0,19-1,87)$ \\
\hline$\geq 60 \mathrm{ml} / \mathrm{min} / 1.73 \mathrm{~m}^{2}$ & 40 & 50,6 & 30 & 50,8 & 70 & 77,8 & 0,00 & $\cdots$ \\
\hline Complicaciones crónic & & & & & & & & \\
\hline Microvasculares & 31 & 48,3 & 33 & 51,6 & 64 & 69,6 & 062 & $1,25(0,46-3,40)$ \\
\hline Macrovasculares & 12 & 42,9 & 16 & 57,6 & 28 & 30,4 &, 02 & $\ldots$ \\
\hline
\end{tabular}




\begin{tabular}{|c|c|c|c|c|c|c|c|c|}
\hline \multirow[t]{2}{*}{ Variable } & \multicolumn{2}{|c|}{$\begin{array}{l}\text { Glicemia } \\
\text { elevada }\end{array}$} & \multicolumn{2}{|c|}{$\begin{array}{c}\text { Glicemia } \\
\text { normal }\end{array}$} & \multicolumn{2}{|c|}{ Total } & \multirow[t]{2}{*}{$p\left({ }^{*}\right)$} & \multirow[t]{2}{*}{ OR (IC 95) } \\
\hline & $\mathbf{n}$ & $\%$ & $\mathbf{n}$ & $\%$ & $\mathbf{n}$ & $\%$ & & \\
\hline \multicolumn{9}{|l|}{ Días de hospitalización } \\
\hline$<14$ días & 34 & 50 & 34 & 50 & 68 & 68 & \multirow{2}{*}{1} & $1(0,39-2,52)$ \\
\hline$\geq 14$ días & 16 & 50 & 16 & 50 & 32 & 32 & & $\ldots$ \\
\hline \multicolumn{9}{|c|}{ Medicación previa a la hospitalización } \\
\hline Sí & 25 & 44,6 & 31 & 55,4 & 56 & 56 & \multirow{2}{*}{0,22} & $0,61(0,25-1,46)$ \\
\hline No & 25 & 56,8 & 19 & 43,2 & 44 & 44 & & $\ldots$ \\
\hline \multicolumn{9}{|l|}{ Medicación al alta } \\
\hline Sí & 48 & 53,3 & 42 & 46,7 & 90 & 90 & \multirow{2}{*}{0,045} & $4,57(0,83-45,8)$ \\
\hline No & 2 & 20 & 8 & 80 & 10 & 10 & & $\ldots$ \\
\hline \multicolumn{9}{|l|}{ Tipo de medicación al alta } \\
\hline Solo ADO & 4 & 30,8 & 9 & 69,2 & 13 & 14,4 & \multirow{2}{*}{0,078} & $0,33(0,06-1,33)$ \\
\hline Insulina / Insulina + ADO & 44 & 57,1 & 33 & 42,9 & 77 & 85,6 & & $\ldots$ \\
\hline \multicolumn{9}{|c|}{ Primer control por consultorio externo } \\
\hline$<30$ días & 36 & 48,6 & 38 & 51,4 & 74 & 74 & \multirow{2}{*}{0,64} & $0,81(0,29-2,18)$ \\
\hline$\geq 30$ días & 14 & 53,8 & 12 & 46,2 & 26 & 26 & & $\ldots$ \\
\hline \multicolumn{9}{|l|}{ Cumplimiento de tratamiento } \\
\hline Cumple & 37 & 43 & 49 & 57 & 86 & 86 & \multirow{2}{*}{0,001} & $\ldots$ \\
\hline No cumple & 13 & 92,9 & 1 & 7,1 & 14 & 14 & & $17,2(2,33-746,0)$ \\
\hline \multicolumn{9}{|l|}{ Servicio de hospitalización } \\
\hline Endocrinología & 30 & 49,2 & 31 & 50,8 & 61 & 61 & \multirow{2}{*}{0,83} & $0,91(0,38-2,21)$ \\
\hline Medicina & 20 & 51,3 & 19 & 48,7 & 39 & 39 & & \\
\hline
\end{tabular}

HbA1c: Hemoglobina glicosilada, LDL: Lipoproteína de baja densidad, HTA: Hipertensión arterial. $\left.\mathbf{(}^{*}\right)$ Se realizó corrección de Yates cuando al menos el valor de una frecuencia esperada fue menor que 5.

En el análisis multivariado, las características que permanecieron asociadas a la alteración de la glicemia basal en el primer control posterior a una hospitalización, en pacientes con DM2, fueron los niveles elevados de triglicéridos y el no cumplimiento con el tratamiento prescrito (Tabla 2).

Tabla 2. Análisis multivariado mediante regresión logística binaria de los factores asociados con la alteración de la glicemia basal en el primer control posterior a una hospitalización en pacientes con diabetes mellitus tipo 2

\begin{tabular}{|c|c|c|}
\hline Variables del modelo & $\mathbf{p}$ & OR (IC) \\
\hline \multicolumn{3}{|l|}{ Glicemia al ingreso } \\
\hline$>250 \mathrm{mg} / \mathrm{dl}$ & 0.78 & $2.7(1.19-6.11)$ \\
\hline \multicolumn{3}{|l|}{ Glicemia al alta } \\
\hline$\geq 140 \mathrm{mg} / \mathrm{dl}$ & 0.27 & $3.4(1.51-7.85)$ \\
\hline \multicolumn{3}{|l|}{ HbA1c durante la hospitalización } \\
\hline$>9 \%$ & 0.19 & $2.9(1.13-7.42)$ \\
\hline \multicolumn{3}{|l|}{ Triglicéridos } \\
\hline$\geq 150 \mathrm{mg} / \mathrm{dl}$ & 0.008 & $2.6(1.1-6.3)$ \\
\hline \multicolumn{3}{|l|}{ Tipo de medicación al alta } \\
\hline Insulina /Insulina + ADO & 0.308 & $1.6(1.03-2.52)$ \\
\hline \multicolumn{3}{|l|}{ Cumplimiento de tratamiento } \\
\hline No cumple & 0.044 & $2.15(1.62-2.85)$ \\
\hline
\end{tabular}

p: valor p en el modelo, HbA1c: Hemoglobina glicosilada, OR: Odds ratio ajustado. 


\section{DISCUSIÓN}

La población del estudio se caracterizó por estar conformada en su mayoría por pacientes mujeres, población similar a la estudiada por M. Khattab et al. ${ }^{(12)}$, en la que la mayor prevalencia de pacientes con DM2 también fue de sexo femenino con $67.1 \%$. De igual manera el estudio realizado por Gonzales et al., en un hospital nacional, describe las características clínicas de una población con diagnóstico de DM2 hospitalizada ${ }^{(15)}$, en la que la mayor parte de su población fue de sexo femenino con un $63.2 \%$. Esto indicaría que, a pesar de las diferencias en las prevalencias poblacionales, no existe diferencia en la proporción según sexo en cuanto a población hospitalizada por DM2. En nuestro estudio se observó además que las mujeres tenían peor control glicémico que los varones, sin embargo esta diferencia no presentó significancia estadística; esto coincide en el estudio realizado por Khattab et al. (12). De igual manera en un metanálisis realizado en la India (16), los varones presentaron mejor control comparado con mujeres, pero tampoco fue significativo.

Respecto a la edad se observó que los pacientes con peor control glicémico fueron aquellos comprendidos entre $18 \mathrm{y}$ 40 años (68\%); aunque esta diferencia no fue significativa, el hallazgo concuerda con lo descrito por Juarez et al. (17), cuyo estudio describe que pacientes con edad < 35 años presentan peor control de la DM2. De igual manera en el metanálisis ya mencionado ${ }^{(16)}$, el control de la diabetes fue peor en los pacientes menores de 60 años en comparación con los mayores (O.R.: 1.61, IC: 1.11 - 2.33). Así mismo el estudio realizado por Feldman et al. se encontró que los subgrupos más vulnerables para el mal control de la glicemia fueron los pacientes con edad < 55 años ${ }^{(18)}$. Otro estudio reciente en los Estados Unidos informó tasas más pobres de adherencia a la medicación entre las edades de 18-64 años, sin embargo esto fue evaluado por autoreporte (19). La alteración de la glicemia basal en el primer control posterior a una hospitalización en el grupo de menor edad podría ser explicada por la falta de adherencia al tratamiento en este grupo etario, como ya lo describen otros estudios.

Nuestro estudio mostró también que el mayor tiempo de enfermedad no está asociado con la alteración de la glicemia basal en el primer control posterior a una hospitalización. Este hallazgo no es consistente con otros reportes donde se asocia el mayor tiempo de enfermedad (> 7 años) con el mal control glicémico ${ }^{(12)}$. De igual modo, otros estudios como el de Benoit et al. (20) y Verma et al. (21), concluyeron que a mayor tiempo de enfermedad, peor control de la glicemia. Es conocido que el deterioro progresivo de la secreción de insulina por el fracaso de las células beta en combinación con la resistencia a la insulina, produzca un empeoramiento del control de la glucosa en el tiempo ${ }^{(19,20)}$. Las poblaciones analizadas en estos estudios son mucho mayores a la nuestra, quizás esa sea la principal razón por la que en nuestro estudio la variable no tuvo significancia estadística.

En el análisis bivariado se encontró que aquellos pacientes que ingresan a hospitalización con glicemia $>250 \mathrm{mg} / \mathrm{dL}$ presentaron mayor riesgo de la alteración de la glicemia basal en el primer control posterior a la hospitalización (OR: 2.7, IC: 1.19 - 6.11), siendo un factor de riesgo significativo. Este resultado no ha sido reportado en estudios similares que hayan analizado la glicemia posterior a la hospitalización.

Así mismo, la glicemia al alta ( $\geq 140 \mathrm{mg} / \mathrm{dL}$ ) también resultó ser un factor de riesgo significativo para el mal control glicémico posterior (OR: 3.7, IC: 1.51-7.85). Este hallazgo demuestra la importancia de lograr los objetivos establecidos para los pacientes hospitalizados, mediante esquemas de tratamiento para cada paciente durante la hospitalización hasta el momento del alta. El no alcanzarlos repercute directamente de forma negativa en el control ambulatorio luego de la hospitalización.

Es importante señalar que, para fines específicos de este estudio, la HbA1c como marcador de buen control glicémico no fue considerado por el tipo de pacientes analizados, quienes habiendo estado hospitalizados recientemente necesariamente presentarían una elevación de la HbA1c; además la glicemia basal es la prueba que nos proporciona de forma inmediata una idea de la situación del paciente al llegar a la consulta. Es evidente, sin embargo y como ya está demostrado ${ }^{(22,23)}$, la necesidad de seguir a los pacientes con un control de HbA1c periódico. La muestra de nuestro estudio presentó valores de HbA1c durante la hospitalización $>9 \%$ en el $56.7 \%$ de pacientes. En el estudio realizado por Feldman et al. que también tomó dicho punto de corte para HbA1c, el $83.3 \%$ estuvo en dicha categoría ${ }^{(17)}$. Valores muy elevados de HbA1c influencia notablemente en el adecuado control de la glicemia posterior a la hospitalización.

En cuanto al manejo farmacológico indicado al alta, en nuestro estudio el tratamiento a base de insulina sola o en combinación resultó ser factor de riesgo para la alteración de la glicemia basal en el primer control posterior a una hospitalización, esto concuerda con otros resultados obtenidos, donde también hubo un mal control de la glicemia en aquellos que usaron insulina, frente a los que no la usaron (1). Un estudio realizado en Jordania mostró de igual modo que la insulina en combinación con antidiabéticos orales se asoció con peor control glicémico (19). Basados en los datos de nuestro estudio y la evidencia adicional encontrada, creemos que aquellos pacientes a los que se les indica terapia de insulina sola o combinado con antidiabéticos orales, podrían tener posteriormente un mal control de glicemia debido a la falta de adherencia 
al tratamiento y a la escasa consejería brindada sobre el modo de administración del medicamento, poca información del beneficio que brinda el uso de la insulina, y el poco conocimiento del riesgo que implica dejar de usar la terapia instaurada.

En relación a la hipertrigliceridemia, debemos tener en cuenta que la DM2 se consideró inicialmente una enfermedad producida por alteración de los carbohidratos, pero actualmente se describe como un trastorno multifactorial con alteraciones asociadas además al metabolismo de proteínas y lípidos ${ }^{(24)}$. La dislipidemia en la DM2 juega un papel importante, siendo más aterogénica que en los pacientes no diabéticos ${ }^{(25)}$. De ahí la importancia de un adecuado control.

En varios estudios, se describió a la dislipidemia como factor de riesgo de mal control de diabetes ${ }^{(1,12,17)}$, aunque en el último de ellos sólo fue significativo un valor de colesterol total $\geq 200$. En nuestro estudio, probablemente por la imposibilidad de analizar el colesterol HDL, no fue significativa la dislipidemia como factor para un mal control glicémico. En cambio, la presencia de triglicéridos $>150 \mathrm{mg} / \mathrm{dL}$ resultó ser un claro factor de riesgo (OR: 2.6, IC: 1.1 - 6.3), datos similares al estudio realizado por Yonas Mullugeta et al., donde se evidenció una correlación significativa (coeficiente de correlación $r$ : $0.28, p<0.05$ ) en relación a los niveles de triglicéridos y el mal control glicémico. La evidencia demuestra que la hipertrigliceridemia es la alteración más frecuente en los pacientes diabéticos y aún más en los mal controlados (24).

Se encontró también que no cumplir con el tratamiento prescrito en el alta hospitalaria es un factor de riesgo significativo ( $p<0.005$, OR : 2.15 IC:1.62 - 2.85), diversos estudios han concluido datos similares, confirmando que existe una fuerte asociación inversa entre adherencia a la medicación y el mal control glicémico, siendo casi tres veces más probable tener una glicemia basal elevada en los pacientes no adherentes al tratamiento, comparados a los que si se adhieren ${ }^{(18)}$. En el trabajo realizado por Khattab et al. ${ }^{(12)}$, observamos resultados similares. Esto traduce la importancia de motivar a los pacientes para utilizar los medicamentos prescritos, además de fomentar la actividad física y una alimentación adecuada.

Los esfuerzos para lograr un buen control de la glicemia en pacientes hospitalizados se ven muchas veces obstaculizados, la duración de la estancia hospitalaria de los pacientes con diabetes se ha ido reduciendo y esta menor estancia hospitalaria no siempre proporciona suficiente tiempo para optimizar el control glicémico cuando otros problemas agudos se están manejando simultáneamente. Una transición eficaz debería incluir un adecuado entrenamiento en habilidades necesarias durante la hospitalización y con un plan adecuado de atención primaria posterior al alta, que en nuestro país aún no es del todo posible por múltiples falencias en el sistema sanitario.

Nuestro estudio tiene las limitaciones de ser un estudio retrospectivo, por lo que algunas variables no pudieron ser incluidas en el análisis de los datos, dentro de ellas están el índice de masa corporal, HDL, contar o no con seguro de salud, entre otras, que podrían relacionarse al mal control glicémico posterior a la hospitalización. Otra limitación fue el seguimiento de los pacientes, siendo importante valorar el seguimiento posterior en estos pacientes. Recomendamos realizar estudios prospectivos donde se consideren estas otras variables.

En conclusión, los niveles de triglicéridos $\geq 150 \mathrm{mg} / \mathrm{dl}$ y el no cumplimiento del tratamiento, son factores de riesgo asociados a un pobre control glicémico en la primera consulta externa luego de la hospitalización; sin embargo, no descartamos los factores encontrados en el análisis bivariado y recomendamos tenerlos en cuenta para el tratamiento del paciente en el área hospitalaria.

La hospitalización debe representar una oportunidad para el equipo de salud para informar a los pacientes sobre el autocontrol de la diabetes después del alta, y los programas de educación pueden incluir proporcionar instrucciones sobre las técnicas de administración de insulina, abordar las preocupaciones de los pacientes acerca de continuar el uso de insulina, visitas en el tiempo necesario para un adecuado seguimiento del paciente ambulatorio con el médico de atención primaria, endocrinólogo, o un educador en diabetes; esto permitiría al paciente, comprender más como controlar su enfermedad y cumplir con los objetivos del tratamiento.

\section{REFERENCIAS BIBLIOGRÁFICAS}

1. Pérez A, Franch J, Cases A, González Juanatey JR, Conthe P, Gimeno E, et al. Relationship between the degree of glycemic control and diabetes characteristics and hyperglycemia treatment in type 2 diabetes. DIABES Study. Med Clin. 2012;138(12):505-11. Disponible en: https://www.ncbi.nlm. nih.gov/pubmed/22118974

2. American Diabetes Association. (4) Foundations of care: education, nutrition, physical activity, smoking cessation, psychosocial care, and immunization. Diabetes Care. 2015;38(1):20-30. Disponible en: https://www.ncbi.nlm.nih. gov/pubmed/25537702

3. Rydén L, Standl E, Bartnik M, Van den Berghe G, Betteridge $\mathrm{J}$, de Boer $\mathrm{M}-\mathrm{J}$, et al. Guidelines on diabetes, pre-diabetes, and cardiovascular diseases: executive summary. Eur Heart J. 2007;28(1):88-136. Disponible en: https://www.ncbi.nlm. nih.gov/pubmed/17220161

4. International Diabetes Federation. IDF Diabetes Atlas Eight Edition. 2017 [Internet]. Disponible en: http://www. diabetesatlas.org/

5. Seclen SN, Rosas ME, Arias AJ, Medina CA. Elevated incidence rates of diabetes in Peru: report from PERUDIAB, a national urban population-based longitudinal study. BMJ Open Diabetes 
Res Care. 2017;5(1):e000401. Disponible en: https://www. ncbi.nlm.nih.gov/pubmed/28878935

6. Cook CB, Seifert KM, Hull BP, Hovan MJ, Charles JC, MillerCage V, et al. Inpatient to outpatient transfer of diabetes care: planing for an effective hospital discharge. Endocr Pract. 2009;15(3):263-9. Disponible en: https://www.ncbi. nlm.nih.gov/pubmed/19364697

7. Jiang HJ, Stryer D, Friedman B, Andrews R. Multiple hospitalizations for patients with diabetes. Diabetes Care. 2003;26(5):1421-6. Disponible en. https://www.ncbi.nlm. nih.gov/pubmed/12716799

8. Ministerio de Salud del Perú. Principales causas de morbilidad de hospitalización por sexo [Internet], Perú, 2015. Disponible en: http://www.minsa.gob.pe/estadisticas/estadisticas/ Morbilidad/HSMacros.asp?00

9. Moghissi ES, Korytkowski MT, DiNardo M, Einhom D, Hellman R, Hirsh IB, et al. American Association of Clinical Endocrinologists and American Diabetes Association Consensus Statement on Inpatient Glycemic Control. Diabetes Care. 2009; 32(6):11191131. Disponible en: https://www.ncbi.nlm.nih.gov/pmc/ articles/PMC2681039/

10. American Diabetes Association. Standards of Medical Care in Diabetes-2011. Diabetes Care. 2011; 34(1):11-61. Disponible en: https://www.ncbi.nlm.nih.gov/pmc/articles/ PMC3006050/

11. Jansen S, Buonaiuto V, Márquez-Gómez I, Gómez-Huelgas R. Management of hyperglycemia in the non-critical hospitalized patients with oral feeding. Rev Clin Esp. 2012; 212(2):84-9. Disponible en: https://www.ncbi.nlm.nih.gov/ pubmed/21798530

12. Khattab M, Khader YS, Al-Khawaldeh A, Ajlouni K. Factors associated with poor glycemic control among patients with type 2 diabetes. J Diabetes Complications. 2010; 24(2):84-9. Disponible en: https://www.ncbi.nlm.nih.gov/ pubmed/19282203

13. Ramos Marini MR. Actualización en el manejo de la cetoacidosis diabética y el estado hiperosmolar hiperglucémico en adultos. Rev Med Hondur. 2011; 79(2):85-93. Disponible en: http:// www.bvs.hn/RMH/pdf/2011/pdf/Vol79-2-2011-10.pdf

14. Handelsman Y, Bloomgarden ZT, Grunberger G, Umpierrez G, Zimmerman RS, Bailey TS, et al. American association of clinical endocrinologists and american college of endocrinology - clinical practice guidelines for developing a diabetes mellitus comprehensive care plan - 2015. Endocr Pract. 2015;21 (1):1-87. Disponible en: https://www.ncbi. $\mathrm{nlm}$.nih.gov/pubmed/25869408

15. Gonzales N, Rodríguez E, Manrique H. Características clínicas y factores asociados a morbilidad intrahospitalaria en los pacientes con diabetes mellitus tipo 2. Rev Soc Peru Med Interna. 2013; 26(4):159-65. Disponible en: http:// medicinainterna.net.pe/pdf/2013/vol26num4/trabajo\%20 original2.pdf

16. Sanal TS, Nair NS, Adhikari P. Factors associated with poor control of type 2 diabetes mellitus : A systematic review and Meta-analysis. J Diabetol. 2011;2(3):4. Disponible en: http:// www. journalofdiabetology.org/article. asp?issn=2078-7685; ye ar $=2011$; volume $=2$; issue $=3$; spage $=4$; epage $=4$; aulast $=$ Sanal; ty pe $=0$

17. Juarez DT. Factors Associated With Poor Glycemic Control or Wide Glycemic Variability Among Diabetes Patients in Hawaii, 2006-2009. Prev Chronic Dis. 2012;9: 120065. Disponible en: https: //www.ncbi.nlm.nih.gov/pubmed/23017247

18. Feldman BS, Cohen-Stavi CJ, Leibowitz M, Hoshen MB, Singer $\mathrm{SR}$, Bitterman $\mathrm{H}$, et al. Defining the role of medication adherence in poor glycemic control among a general adult population with diabetes. PLoS One. 2014;9(9): e108145.Disponible en: http://journals.plos.org/plosone/ article?id=10.1371/ journal. pone. 0108145
19. Lopez JMS, Bailey RA, Rupnow MFT, Annunziata K. Characterization of type 2 diabetes mellitus burden by age and ethnic groups based on a nationwide survey. Clin Ther. 2014;36(4):494-506. Disponible en: http://www. clinicaltherapeutics.com/article/S0149-2918(14)00013-7/ abstract

20. Benoit SR, Fleming R, Philis-Tsimikas A, Ji M. Predictors of glycemic control among patients with Type 2 diabetes: A longitudinal study. BMC Public Health. 2005;5 (1):36. Disponible en: https://bmcpublichealth.biomedcentral.com/ articles/10.1186/1471-2458-5-36

21. Verma M., Paneri S., Raman P.G. Effect of increasing duration of diabetes mellitus type 2 on glycated hemoglobin and insulin sensitivity. Indian J Clin Biochem. 2006;21(1):142-6. Disponible en: https://link.springer.com/article/10.1007/ BF02913083

22. Group UPDS. Intensive blood-glucose control with sulphonylureas or insulin compared with conventional treatment and risk of complications in patients with type 2 diabetes. The Lancet. 1998;352(9131):837-53. Disponible en: http://www.thelancet.com/journals/lancet/article/ PIIS0140-6736(98)07019-6/abstract

23. Williams Textbook of Endocrinology - 12th Edition [Internet]. [citado 21 de agosto de 2017]. Disponible en: https://www. elsevier.com/books/williams-textbook-of-endocrinology/ melmed/978-1-4377-0324-5

24. Mullugeta Y, Chawla R, Kebede T, Worku Y. Dyslipidemia Associated with Poor Glycemic Control in Type 2 Diabetes Mellitus and the Protective Effect of Metformin Supplementation. Indian J Clin Biochem. 2012;27(4):3639. Disponible en: https://www.ncbi.nlm.nih.gov/ pubmed $/ 24082461$

25. Krauss RM. Lipids and lipoproteins in patients with type 2 diabetes. Diabetes Care. 2004;27(6):1496-504. Disponible en: https://www.ncbi.nlm.nih.gov/pubmed/15161808

Fuentes de financiamiento:

Este artículo ha sido financiado por los autores.

Conflictos de interés:

Los autores declaran no tener ningún conflicto de interés.

\section{Correspondencia:}

Ana Denisse Alva Cabrera

Dirección: Jirón Granada 385 - dpto. 402- Pueblo Libre

Teléfono: 995515892

Correo electrónico: denisse_dac@hotmail.com

Recibido: 05 de marzo de 2018

Evaluado: 07 de marzo de 208

Aprobado: 23 de marzo de 2018

(C) La revista. Publicado por Universidad de San Martín de Porres, Perú. (cc) ву Licencia de Creative Commons Artículo en acceso abierto bajo términos de Licencia Creative Commons Atribución 4.0 Internacional. (http: //creativecommons.org/licenses/by/4.0/)

ORCID iDs

Ana Denisse Alva Cabrera Wilfor Aguirre Quispe Carlos Alexander Alva Díaz Alexander Amilcar Zapana Mansilla Jorge Alonso García Mostajo
iD https://orcid.org/0000-0001-5464-1629 D https://orcid.org/0000-0002-6677-0900 https://orcid.org/0000-0003-3584-7298 https://orcid.org/0000-0003-3124-0253 https://orcid.org/0000-0002-8073-0008 